\title{
Atomic force microscopy at ambient and liquid conditions with stiff sensors and small amplitudes
}

\author{
Elisabeth Wutscher ${ }^{\mathrm{a})}$ and Franz J. Giessibl \\ Institute of Experimental and Applied Physics, University of Regensburg, 93040 Regensburg, Germany
}

(Received 17 June 2011; accepted 15 August 2011; published online 12 September 2011)

\begin{abstract}
We report on atomic force microscopy (AFM) in ambient and liquid environments with the qPlus sensor, a force sensor based on a quartz tuning fork with an all-electrical deflection measurement scheme. Small amplitudes, stiff sensors with bulk diamond tips and high $Q$ values in air and liquid allow to obtain high resolution images. The noise sources in air and liquid are analyzed and compared for standard silicon cantilevers and qPlus sensors. First, epitaxial graphene was imaged in air, showing atomic steps with $3 \AA$ height and ridges. As a second sample system, measurements on calcite $\left(\mathrm{CaCO}_{3}\right)$ in liquids were performed in water and polyethylenglycol (PEG). We demonstrate high resolution images of steps in PEG on calcite and nanolithography processes, in particular with frequency-modulation AFM the controlled dissolution of calcite monolayers. (C) 2011 American Institute of Physics. [doi:10.1063/1.3633950]
\end{abstract}

\section{INTRODUCTION}

In the last years, high resolution images on the atomic scale with frequency-modulation atomic force microscopy (FM-AFM) in ambient conditions were achieved. Fukuma et al. ${ }^{1}$ succeeded in resolving true atomic resolution on the (001) surface of muscovite mica in water, recorded with small amplitudes and silicon cantilevers with an eigenfrequency $f_{0} \approx 150 \mathrm{kHz}$ and stiffness $k \approx 40 \mathrm{~N} / \mathrm{m}$. FM-AFM has initially been the method of choice for high resolution AFM in ultra-high-vacuum (UHV).

There also impressive results have been shown recently at room- and low-temperature with FM-AFM. With the qPlus sensor, a force sensor based on a quartz tuning fork ${ }^{2,3}$ $\left(f_{0} \approx 30 \mathrm{kHz}, k \approx 1800 \mathrm{~N} / \mathrm{m}\right)$, subatomic features on $\operatorname{Si}(111)$ $7 \times 7$ were imaged. ${ }^{4}$ Since then it has been used to measure the force it takes to move an atom over a surface ${ }^{5}$ and to resolve the chemical structure of a molecule. ${ }^{6}$ This success could be reached by utilizing the high stiffness of the qPlus sensor and small amplitudes. Because the concept of the stiff sensors and the small amplitudes enhanced the force resolution in UHV, it is expected to also improve resolution in ambient conditions.

Here, we report on measurements taken at ambient and liquid conditions with a qPlus sensor. At first the noise sources in FM-AFM at ambient conditions are calculated. The detectable force limits of standard cantilevers used in liquid ${ }^{1}$ are compared with the stiffer qPlus sensor. First data in air and liquid with the qPlus sensor at small amplitudes are shown.

\section{LIMITS OF FORCE RESOLUTION: NOISE CONTRIBUTIONS}

In FM-AFM a sensor with a stiffness $k$ oscillates at its resonance frequency $f_{0}$ close to a sample surface. Due to the interaction between tip and sample, which can be described by the average force gradient $\left\langle k_{t s}\right\rangle$, the oscillation frequency

\footnotetext{
a)Electronic mail: elisabeth.wutscher@physik.uni-r.de.
}

of the sensor changes to $f=f_{0}+\Delta f$. The frequency shift is $^{7}$

$$
\Delta f=\left\langle k_{t s}\right\rangle \frac{f_{0}}{2 k}
$$

To achieve the best resolution, we have to analyze the factors that limit the accuracy of our frequency shift measurement. Such noise sources are: thermal noise, detector noise, and oscillator noise, as explained below.

Thermal vibrations of the cantilever, caused by random kicks of the cantilever by its environment, lead to thermal frequency noise. According to Albrecht et al., ${ }^{8}$ the thermal noise density is given by

$$
n_{f t h}=\sqrt{\frac{k_{B} T f_{0}}{\pi k A^{2} Q}} .
$$

It depends on the thermal stored energy $k_{B} T$, the stiffness $k$, the quality factor $Q$, and the amplitude $A$ of the cantilever. Thermal excitation can be used to measure the resonance curve of the cantilever. ${ }^{9}$

In general, the frequency noise density is used to calculate the frequency noise $\delta f$ in a frequency measurement, where

$$
\delta f=\sqrt{\int_{0}^{B} n_{f}^{2} d f} .
$$

An additional noise source is detector noise, caused by the inaccuracy of the deflection measurement with optical or electrical means. The detector noise density is ${ }^{11}$

$$
n_{f d e t}=\frac{\sqrt{2} n_{q}}{A} f_{\text {mod }} .
$$

The term $n_{q}=\frac{n_{e l}}{S}$ describes the deflection noise density, which is given by ratio between the electrical noise density $n_{e l}$ and the sensitivity $S$ of the electrical signal. ${ }^{10}$ These two components can be measured by the thermally excited signal of the cantilever. 
Kobayashi et al. ${ }^{11}$ have identified another noise term, the oscillator noise, which is significant for low $Q$ environments. In the self-oscillation feedback circuit, incoming noise in the phase signal from the cantilever is transferred into noise in the outgoing excitation frequency. This noise source is inversely proportional to the slope of the phase versus frequency shift curve $-2 Q / f_{0}$ at the resonance frequency $f_{0}$. For a fixed phase noise, the frequency noise becomes larger with lower $Q$ values. This results in the oscillator noise density as

$$
n_{f o s c}=\frac{f_{0} n_{q}}{\sqrt{2} Q A} .
$$

Because the three noise sources are independent, they have to be added in quadrature to determine the total frequency noise density,

$$
\begin{aligned}
n_{F M} & =\sqrt{n_{f t h}^{2}+n_{f d e t}^{2}+n_{f o s c}^{2}} \\
& =\sqrt{\frac{k_{B} T f_{0}}{\pi k A^{2} Q}+\frac{2 n_{q}^{2}}{A^{2}} f_{m o d}^{2}+\frac{n_{q}^{2}}{2 Q^{2} A^{2}} f_{0}^{2}} .
\end{aligned}
$$

A fourth source of noise is caused by the thermal dependence of $f_{0}$. When the sensor temperature changes, $f_{0}$ also changes. The rate of change $\delta f_{0} / \delta T$ is a function of the cantilever material. For silicon cantilevers the frequency varies quite strong as a function of temperature, whereas quartz is remarkably stable at room temperature. ${ }^{12}$ This noise source, however, can be minimized for silicon cantilevers by measuring in liquid in an incubator, where the temperature is kept constant at $298 \pm 0.15 \mathrm{~K}^{13}$

In general, the frequency noise density depends on several parameters: the cantilever (characterized by $f_{0}$ and $k$ ), the actual oscillation amplitude $A$, the deflection noise density $n_{q}$ (defined by the measurement setup and the preamplifiers), and the quality factor $Q$ (which is strongly depending on the sensor and the environment like UHV, air or liquid). To optimize the parameters one must take into account, that it is not the frequency noise alone that counts for the scanning resolution, but also the magnitude of the frequency shift signal, given in Eq. (1) by $\delta\left\langle k_{t s}\right\rangle_{\min }=\delta \Delta f \cdot \frac{2 k}{f_{0}}$. The frequency noise density has to be first integrated over the modulation frequency to get the frequency noise, ${ }^{12}$

$$
\begin{aligned}
\delta \Delta f & =\sqrt{\int_{0}^{B} n_{F M}^{2} d f_{\text {mod }}} \\
& =\sqrt{\frac{k_{B} T f_{0} B}{\pi k A^{2} Q}+\frac{2 n_{q}^{2}}{3 A^{2}} B^{3}+\frac{n_{q}^{2} f_{0}^{2}}{2 Q^{2} A^{2}} B .}
\end{aligned}
$$

The frequency noise is the change in the measured frequency shift $\delta \Delta f$ with the FM bandwidth $B$, which yields the minimum detectable average force gradient $\delta\left\langle k_{t s}\right\rangle_{\text {min }}$. In the same manner also the force gradient for the thermal-, detector-, and oscillator frequency noise can be calculated:

$$
\delta k_{t s t h}=2 k \frac{\delta f_{t h}}{f_{0}}=\sqrt{\frac{4 k k_{B} T B}{\pi f_{0} A^{2} Q}},
$$

TABLE I. The values for the cantilever in liquid are taken from Fukuma et al. (Ref. 1) (except for the bandwidth $B$ which was set to $1 \mathrm{kHz}$ in Fukuma et al.'s atomically resolved data, where $\delta\left\langle k_{t s}\right\rangle_{\min }$ amounts to $25 \mathrm{mN} / \mathrm{m}$ ). The qPlus sensor data are the ones used in the experimental part and the qPlus* data are calculated for a recently tested amplifier with a lower deflection noise density. The force gradient for the thermal- $\left(\delta k_{t s} t h\right)$, detector- $\left(\delta k_{t s} d e t\right)$, and oscillator frequency noise $\left(\delta k_{t s ~ o s c}\right)$ is listed and the minimum detectable average force gradient $\delta\left\langle k_{t s}\right\rangle_{\min }$ can be compared for the different sensors in liquid.

\begin{tabular}{lccc}
\hline \hline Parameter & Cantilever & qPlus & qPlus* \\
\hline$f_{0}(\mathrm{kHz})$ & 136 & 50 & 50 \\
$k(\mathrm{~N} / \mathrm{m})$ & 42 & 4300 & 4300 \\
$A(\mathrm{~nm})$ & 0.3 & 0.3 & 0.3 \\
$Q$ & 30 & 300 & 300 \\
$n_{q}(\mathrm{fm} / \sqrt{\mathrm{Hz}})$ & 17 & 85 & 35 \\
$B$ & 50 & 50 & 50 \\
$\delta k_{t s t h}(\mathrm{mN} / \mathrm{m})$ & 5.46 & 28.8 & 28.8 \\
$\delta k_{t s \text { det }}(\mathrm{mN} / \mathrm{m})$ & 0.0101 & 14.1 & 5.79 \\
$\delta k_{t s \text { osc }}(\mathrm{mN} / \mathrm{m})$ & 0.793 & 40.6 & 16.7 \\
$\delta\left\langle k_{t s}\right\rangle_{\text {min }}(\mathrm{mN} / \mathrm{m})$ & 5.51 & 51.7 & 33.8 \\
\hline \hline
\end{tabular}

$$
\begin{gathered}
\delta k_{t s d e t}=2 k \frac{\delta f_{d e t}}{f_{0}}=\sqrt{\frac{8}{3}} \frac{n_{q} k}{A f_{0}} B^{3 / 2}, \\
\delta k_{t s \text { osc }}=2 k \frac{\delta f_{\text {osc }}}{f_{0}}=\frac{\sqrt{2 B} k n_{q}}{A Q} .
\end{gathered}
$$

The various noise force gradients for a cantilever in liquid $^{1}$ and a qPlus sensor in liquid are listed in Table I. At a bandwidth of $50 \mathrm{~Hz}$, Fukuma et al.'s cantilever in liquid has a minimal detectable force gradient of $5.51 \mathrm{mN} / \mathrm{m}$ and for the qPlus sensor, we find $\delta\left\langle k_{t s}\right\rangle_{\text {min }}=51.7 \mathrm{mN} / \mathrm{m}$. Due to high scan speeds, the actual bandwidth $B$ in the experiments of Fukuma et al. is set to $1000 \mathrm{~Hz}$, where a minimal detectable average force gradient of $25 \mathrm{mN} / \mathrm{m}$ results. In Ref. 1, atomic resolution was obtained at a cantilever with $k=42 \mathrm{~N} / \mathrm{m}$ and $f_{0}=136 \mathrm{kHz}$ at frequency shifts ranging from 250 to $500 \mathrm{~Hz}$ (Fig. 4 in Ref. 1), yielding an average tip-sample force gradient between 155 and $310 \mathrm{mN} / \mathrm{m}$ (see Eq. (1)). Thus, for a signal-to-noise ratio of 10 , a minimal noise of $\sim 30 \mathrm{mN} / \mathrm{m}$ is necessary to obtain true atomic resolution on mica. Therefore we can expect, that atomic resolution in liquid by the qPlus sensor can be reached.

The qPlus sensor's greater stiffness and larger deflection noise are partially offset by its higher $Q$ values in air $(1000$ to 2500) and liquid ( 2300$)$ compared to standard silicon cantilevers in air ( 400) (Ref. 14) and liquid ( 30). Currently, the deflection noise density $n_{q}$ of the cantilever $(17 \mathrm{fm} / \sqrt{\mathrm{Hz}})$ is lower than $n_{q}$ for the qPlus sensor $(85 \mathrm{fm} / \sqrt{\mathrm{Hz}})$. The deflection noise density $n_{q}$ depends on the geometry of the quartz sensor and the electric characteristics of the amplifiers and we are continuously working on reducing $n_{q}$. A recently tested amplifier reaches a deflection noise density $n_{q}$ of $35 \mathrm{fm} / \sqrt{\mathrm{Hz}}$, which results in a minimal detectable average force gradient of $34 \mathrm{mN} / \mathrm{m}$ (see Table I: qPlus*). With this amplifier, data will be acquired in the future. 

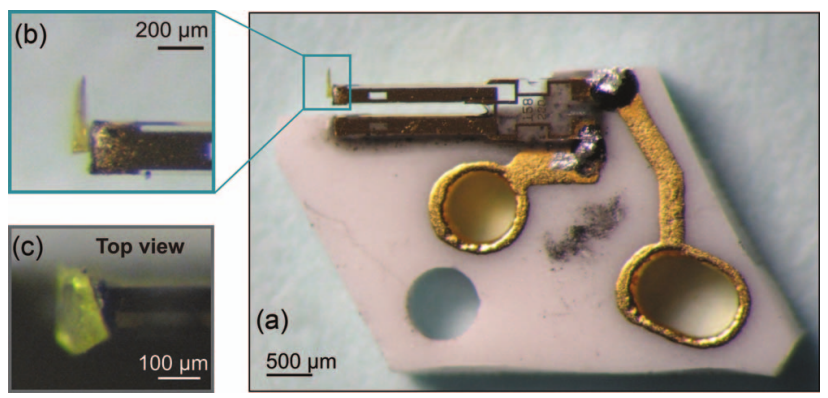

FIG. 1. (Color online) (a) qPlus sensor with glued diamond tip on the front surface of the free tuning fork prong. In (b) the magnification of the tip, and (c) the top view of the tapered diamond tip are depicted.

The dominant noise term for the qPlus sensor in liquid is the oscillator noise $\delta k_{t s ~ o s c}$ (see Eq. (10) and Table I), which scales with the factor $n_{q} / Q$. This means that the disadvantage of high deflection noise densities for the qPlus sensor could be reduced with higher $Q$ values. But while there do exist also the other listed noise sources, the experimental data has to prove if the minimal detectable change in the force gradient is small enough to get atomic resolution with small amplitudes and stiff qPlus sensor in air or liquid.

\section{SENSOR WITH BULK DIAMOND TIP}

The qPlus sensor consists of a shortened E158 tuning fork with a stiffness of $4300 \mathrm{~N} / \mathrm{m}$ and a resonance frequency of around $60 \mathrm{kHz}$. The original E158 tuning fork has a resonance frequency $f_{0}=2^{15} \mathrm{~Hz}$ and a stiffness $k=1800 \mathrm{~N} / \mathrm{m}$, but by cutting off $600 \mu \mathrm{m}$ from these tuning forks at the front end, the resonance frequency and the stiffness increases. ${ }^{15}$ The increase in stiffness is required in ambient conditions to ensure stable oscillations at small amplitudes on soft samples. For standard sensors with $k=1800 \mathrm{~N} / \mathrm{m}$, perturbations by the tip-sample interactions are so strong, that either the amplitude breaks down completely or the resonance frequency shifts about some hundred Hertz and the tuning fork oscillates in a mode with a lower frequency. A short tuning fork glued on an aluminum oxide substrate is shown in Fig. 1(a).

As a base tip material, we choose a hexoctahedral synthetic diamond (De Beers, type SDB1125) with a diameter of
$0.5 \mathrm{~mm}$. After cleaving the bulk diamond with a flat nose plier, a suitable splinter was glued with epoxy (Torr Seal or Loctite Hysol 1C) to the front surface of the tuning fork, as illustrated in Figure 1(a). A magnification of the tip can be seen in Fig. 1(b). The tapered diamond tip is shown from top view in Figure 1(c). Bulk diamond tips were chosen, because in contrast to metal tips, they do not show apparent wear. ${ }^{16}$ For scanning in air and liquids, diamond is most suitable, because it is highly hydrophobic and reduces the capillary forces that act at ambient conditions. ${ }^{17}$

\section{MEASUREMENTS IN AIR ON GRAPHENE}

Graphene samples grown on SiC (Ref. 18) were used for measurements in air. On this sample system, Filleter et al. achieved atomic resolution with FM-AFM in UHV, differentiated between single and double layers with Kelvin probe force microscopy and measured the friction and dissipation. ${ }^{19,20}$

No further preparation (heating or cleaning) was done before scanning the samples. Images were acquired at a resonance frequency $f_{0}=51807 \mathrm{~Hz}$, amplitude $A \approx 6 \AA$ and a frequency shift of $\Delta f=+6 \mathrm{~Hz}$. It is possible to get stable oscillations and images in air with amplitudes down to $3 \AA$.

Steps with heights as small as $3 \AA$ were imaged. Graphene ridges were found at step edges and on terraces, which are marked with arrows in Figures 2(a) and 2(b). The graphene ridges are due to the thermal expansion difference between graphene and $\mathrm{SiC}$ as the samples are rapidly cooled after graphitization. ${ }^{21}$ Panel (a) depicts the raw topography data and Panel (b) is the same image rendered in $3 \mathrm{D}$, with a ten time magnification of the topography in $z$-direction. The area on the left side (the lowest terrace in Fig. 2(b)) has a larger roughness and shows some contamination.

The quality factor of the stiff qPlus sensor in air can be up to 2500 , which would highly improve the resolution. The limiting factor is the preparation of the bulk diamond tip, which does not provide a well-defined sharp tip in every case.

\section{MEASUREMENTS IN LIQUID ON CALCITE}

Measurements in liquid are done to overcome the capillary force which is usually present for all samples exposed to
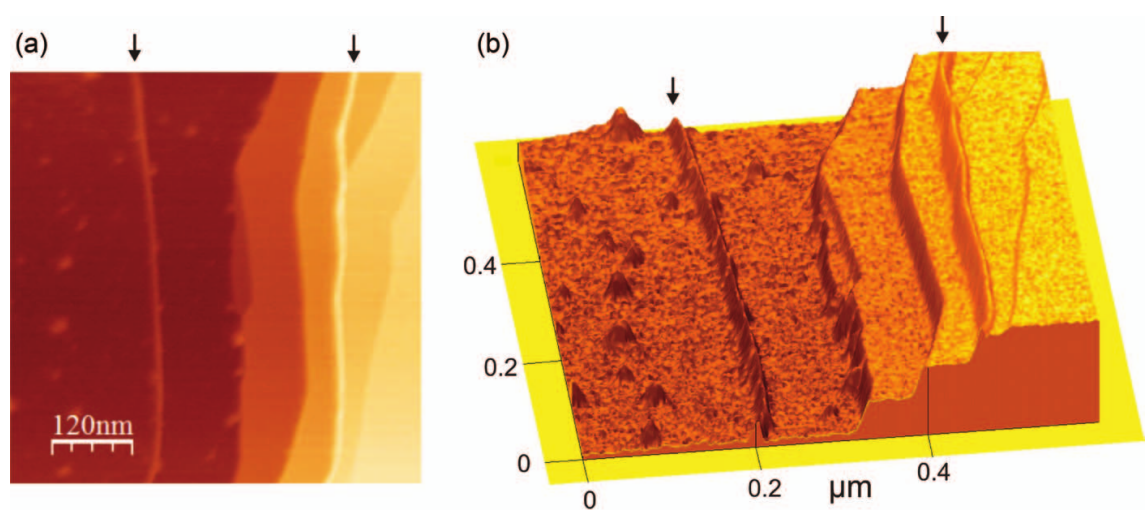

FIG. 2. (Color online) (a) Topography of the graphene surface. (b) Same image rendered in 3D, with a ten time magnification in the $z$-direction to point out the graphene ridges on the lower terrace and on top of the steps. The ridges are marked with arrows in both pictures. 


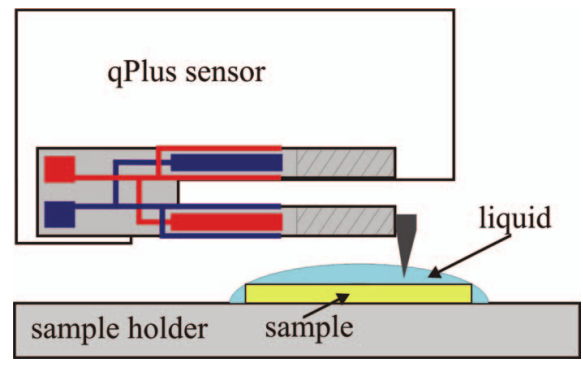

FIG. 3. (Color online) Schematic arrangement of sensor and sample. Only the tip of the qPlus sensor is immersed in liquid, therefore the damping is limited and the $Q$ value stays relatively high.

air and caused by water molecules on the surface. ${ }^{22-24}$ Also the liquid prevents the surface from getting contaminated.

Atomic resolution with quasistatic AFM in liquid was first demonstrated by Ohnesorge and Binnig in 1993 on calcite (1014) in water. ${ }^{25}$ Since then the measurement technics improved and with amplitude-modulation AFM and FMAFM it was also possible to achieve molecular and atomic resolution in liquids. ${ }^{1,13,26}$

Calcite as a sample material is mainly used to observe crystal growth and dissolution processes in liquids. ${ }^{27-32}$ Especially, De Yoreo et al. have shown that it is possible to change the shape of calcite by adding, e.g., magnesium or acidic amino acids to the growth solution of calcite. ${ }^{33,34}$ In this paper we want to image calcite in liquids, because it provides atomic resolution.

In FM-AFM, a high $Q$ value is beneficial (see Eq. (7)), therefore we did not immerse the whole qPlus sensor but only its tip (see Fig. 3). If the liquid were to cover completely the free prong, stable oscillations would not be possible and the electrodes would need to be isolated to prevent leakage currents. This can be avoided by using a small amount of liquid, just sufficient to cover the surface. The additional mass on the tip due to the liquid causes a negative frequency shift. During the approach process, this shift continuously decreases until the sample surface is reached. Conversely, a retraction of the sensor in $z$-direction (about $300 \mathrm{~nm}$ ) causes changes of some hundred $\mathrm{Hz}$ in the frequency shift. These problems can be overcome by controlling the feedback on the damping, which increases with increasing mass of the liquid.
At first, we used a small amount of purified water - just enough to cover completely the calcite sample. By using just a small drop of water, which covers only the top of the sample, the water cover evaporates in a short time, before the tip reaches the sample. With larger amounts of water, as soon as the tip penetrated the water surface layer, the water covered the tip completely, as well as parts of the sensor due to the high surface tension. Then water has shortened the electrodes of the sensor. To overcome these problems we used a liquid with a much lower evaporation rate: polyethylenglycol (PEG) 600 , for all experiments with calcite. PEG 600 (CAS number: 25322-68-3) has a much lower vapor pressure (1.32 Pa) with a lower surface tension (45.76 $\mathrm{mN} / \mathrm{m}$, Ref. 35) than water (3.2 $\mathrm{kPa}$ and $70 \mathrm{mN} / \mathrm{m}$ at $25^{\circ} \mathrm{C}$ ) and is suited very well for this purpose.

Natural calcite is freshly cleaved with a razor blade along the preferred (10 $\overline{1} 4)$ cleavage plane. After that it is glued onto a sample holder and covered with PEG. Steps are $3.16 \AA$ A high and the atomic spacing within a lattice constant of $|\mathbf{a}|=8.1 \AA$ and $|\mathbf{b}|=5.0 \AA$ for the protruding oxygen atoms. ${ }^{25}$

Measurements were taken in PEG on calcite. The images shown in Figure 4 were taken at a resonance frequency $f_{0}$ $=49978 \mathrm{~Hz}$, an amplitude $A=9 \AA$, and the frequency shift $\Delta f=+10 \mathrm{~Hz}$. As mentioned earlier, scanning in liquid lowers the resonance frequency. The resonance frequency of the tuning fork outside the liquid was $51418 \mathrm{~Hz}$ and $1440 \mathrm{~Hz}$ lower inside the PEG. Several steps with a height of $3.16 \AA$ were resolved.

It was possible to dissolve monolayers of the calcite into the PEG with the same sensor caused by a change of the setpoint to $+40 \mathrm{~Hz}$. Figure 5 shows a progression of scans. Figure 5(a) shows the first image of $105 \mathrm{~nm} \times 105 \mathrm{~nm}$, Fig. 5(b) was taken at the same center at $200 \mathrm{~nm} \times 200 \mathrm{~nm}$. The square depression in the center of the image is the size of the scan shown in Fig. 5(a). Over this area, one monolayer of calcite was removed. The further panels of Figs. 5(c) and 5(d) are subsequent and show similar features. Deep horizontal lines are caused by scanning the baseline of new scanframes. By measuring the width of single dissolved scanlines, the tip size was determined to be smaller than $7 \mathrm{~nm}$.

Pits, like the two in the middle of Figure 5(a), are corrosion centers which lead to an enlarged damage after several scans. These areas show dissolved areas deeper than one monolayer; the former pointlike spots enlarged to large pits.
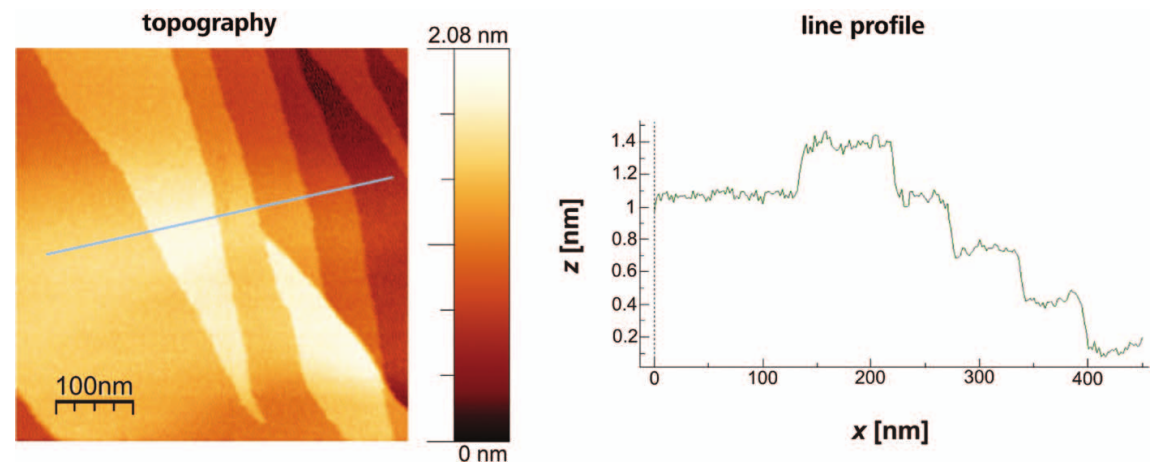

FIG. 4. (Color online) Topographic image of calcite, showing steps in PEG. A profile line in the topography depicts steps with a height of $3.16 \AA$. 

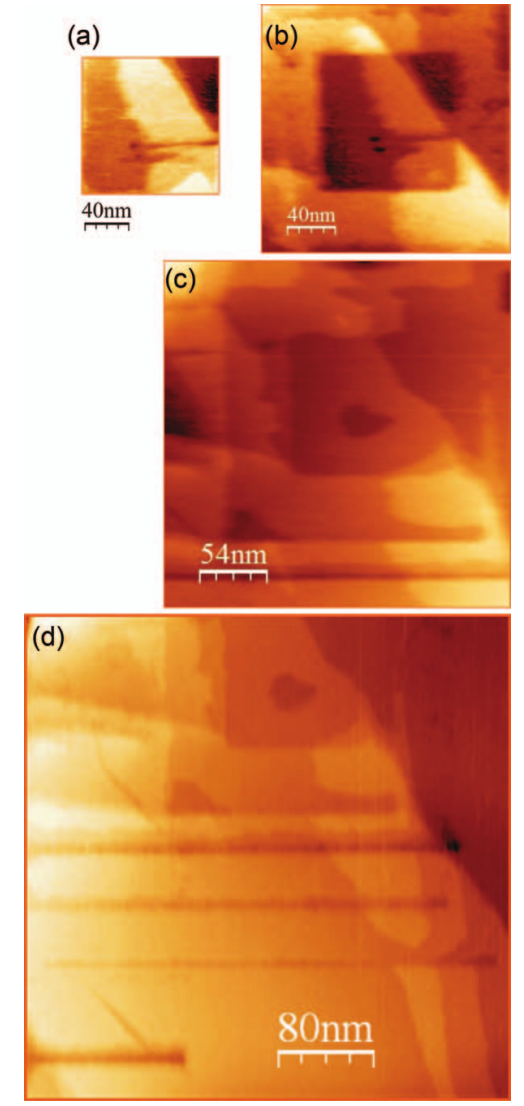

FIG. 5. (Color online) Progression of scans showing continuous dissolution process on calcite. The dissolution is caused by an increased setpoint of the frequency shift (from $+10 \mathrm{~Hz}$ to $+40 \mathrm{~Hz}$ ). Picture (a) with a size of $105 \mathrm{~nm}$ depicts the center for the series. For (b) the size was increased to $200 \mathrm{~nm}$. Clearly the dissolution of one monolayer of the calcite surface can been examined. In (c) the center was moved slightly to the right and upwards. Here, larger dissolved areas can be seen. The bar in picture (a) from the bottom right to the top left is now completely gone. Picture (d) has the center on the top of the scanframe. Horizontal lines are caused by scans of the baseline before starting a new scan.

Our dissolution process is obviously assisted by energy transfer between the tip and the sample. The stored energy from the cantilever is carried over to the calcite and leads to an increased dissolution rate. We believe that the tip-assisted dissolution does not lead to releasing clusters from the sample material, but only ions because we did not observe any debris in a subsequent larger scanframe. The dissolution process could be stopped by changing the $\Delta f$ setpoint back to $+10 \mathrm{~Hz}$ and high quality imaging was again possible.

Processes like the one shown here can be viewed as mechanical AFM lithography. These methods are based on mechanically engraving soft surfaces with AFM tips for further lift-off steps. ${ }^{36,37}$ But a nanolithography process, where just a monolayer of the sample is dissolved by FM-AFM, has not been reported yet.

As well as flat terraces and straight step edges, different structures have been resolved on the calcite surface. In Figure 6, meandering step edges can be observed on the calcite surface. The imaging parameters are $f_{0}=49897 \mathrm{~Hz}$, amplitude $A=3 \AA$, and a frequency shift of $\Delta f=+16 \mathrm{~Hz}$.
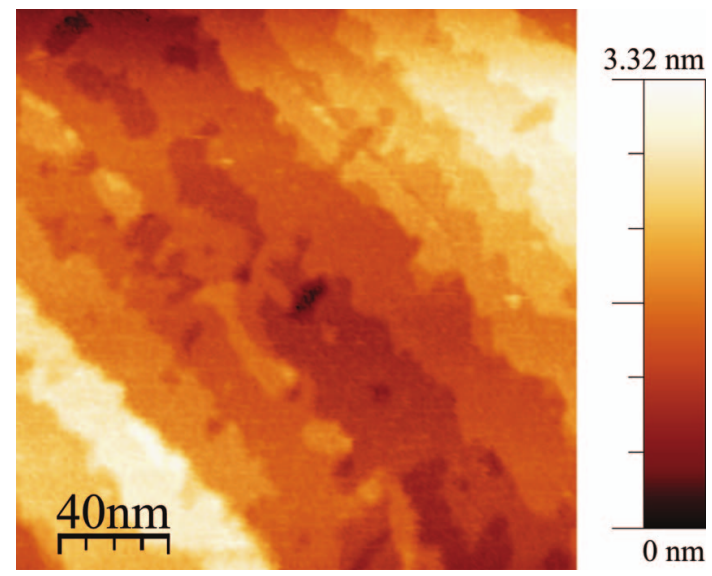

FIG. 6. (Color online) Topography of a different kind of surface structure on calcite. Meandering step edges can be seen.

\section{CONCLUSION}

The concept of imaging with stiff cantilever and small amplitudes, by using the qPlus sensor succeeded in air as well as in liquid. The qPlus sensor outfitted with bulk diamond tips provides high-quality images where the tips do not show apparent wear and a simple electrical readout scheme allows to perform force microscopy without a complicated optical redout scheme. This enables AFM measurements on samples that should not be exposed to light. High resolution images of epitaxial graphene in air with ridges were presented. In PEG calcite monoatomic steps were shown and also nanolithography processes with small tip radii were demonstrated. The advantage of using high $Q$ values to image in air and liquid, allows to obtain high resolution images, but not atomic resolution yet. The comparison in Table I shows that our sensor noise should be sufficiently low to obtain true atomic resolution. Possibly, our sample preparation technique or the properties of the imaging liquid are not yet suitable to succeed on atomic resolution. However, we are investigating other sample and tip systems and we are also working on further reduction of $n_{q}$ by improving our amplifiers. So we are confident that the stiff cantilever/small amplitude technique, that has been so successful in vacuum, will soon provide atomic resolution in ambient conditions as well.

\section{ACKNOWLEDGMENTS}

We like to thank the Graduiertenkolleg 1570 "Electronic Properties of Carbon Based Nanostructures" for financial support, Thomas Seyller and Florian Speck from the University of Erlangen for supplying the graphene samples, A. J. Weymouth for editorial suggestions, and Thorsten Wutscher for his support.

\footnotetext{
${ }^{1}$ T. Fukuma, K. Kobayashi, K. Matsushige, and H. Yamada, Appl. Phys. Lett. 87, 034101 (2005)

${ }^{2}$ F. J. Giessibl, Appl. Phys. Lett. 73, 3956 (1998).

${ }^{3}$ F. J. Giessibl, Appl. Phys. Lett. 76, 1470 (2000).

${ }^{4}$ F. J. Giessibl, S. Hembacher, H. Bielefeldt, and J. Mannhart, Science 289,
} 422 (2000). 
${ }^{5}$ M. Ternes, C. P. Lutz, C. F. Hirjibehedin, F. J. Giessibl, and A. J. Heinrich, Science 319, 1066 (2008).

${ }^{6}$ L. Gross, F. Mohn, N. Moll, P. Liljeroth, and G. Meyer, Science 325, 1110 (2009).

${ }^{7}$ F. J. Giessibl, Appl. Phys. Lett. 78, 123 (2001).

${ }^{8}$ T. R. Albrecht, P. Grütter, D. Horne, and D. Rugar, J. Appl. Phys. 69, 668 (1991).

${ }^{9}$ T. Fukuma and S. P. Jarvis, Rev. Sci. Instrum. 77, 043701 (2006).

${ }^{10}$ S. Hembacher, F. J. Giessibl, and J. Mannhart, Appl. Surf. Sci. 188, 445 (2002).

${ }^{11}$ K. Kobayashi, H. Yamada, and K. Matsushige, Rev. Sci. Instrum. 80, 043708 (2009).

${ }^{12}$ F. J. Giessibl, Rev. Mod. Phys. 75, 949 (2003).

${ }^{13}$ S. Rode, N. Oyabu, K. Kobayashi, H. Yamada, and A. Kühnle, Langmuir 25, 2850 (2009).

${ }^{14}$ T. Fukuma, T. Ichii, K. Kobayashi, H. Yamada, and K. Matsushige, Appl. Phys. Lett. 86, 034103 (2005).

${ }^{15}$ M. Schmid, J. Mannhart, and F. J. Giessibl, Phys. Rev. B 77, 045402 (2008).

${ }^{16}$ E. Oesterschulze, W. Scholz, Ch. Mihalcea, D. Albert, B. Sobisch, and W. Kulisch, Appl. Phys. Lett. 70, 435 (1997).

${ }^{17}$ A. Olbrich, B. Ebersberger, C. Boit, P. Niedermann, W. Hänni, J. Vancea, and H. Hoffmann, J. Vac. Sci. Technol. B 17, 1570 (1999).

${ }^{18}$ K. V. Emtsev, A. Bostwick, K. Horn, J. Jobst, G. L. Kellogg, L. Ley, J. L. McChesney, T. Ohta, S. A. Reshanov, J. Röhrl, E. Rotenberg, A. K. Schmid, D. Waldmann, H. B. Weber, and T. Seyller, Nature Mater. 8, 203 (2009).

${ }^{19}$ T. Filleter, K. V. Emtsev, T. Seyller, and R. Bennewitz, Appl. Phys. Lett. 93, 133117 (2008).
${ }^{20}$ T. Filleter, J. L. McChesney, A. Bostwick, E. Rotenberg, K. V. Emtsev, T. Seyller, K. Horn, and R. Bennewitz, Phys. Rev. Lett. 102, 086102 (2009).

${ }^{21}$ J. Hass, W. A. de Heer, and E. H. Conrad, J. Phys. 20, 323202 (2008).

${ }^{22}$ A. L. Weisenhorn, P. K. Hansma, T. R. Albrecht, and C. F. Quate, Appl. Phys. Lett. 54, 2651 (1989).

${ }^{23}$ Y. Gan, Surf. Sci. Rep 64, 99 (2009).

${ }^{24}$ M. Henderson, Surf. Sci. Rep. 46, 1 (2002).

${ }^{25}$ F. Ohnesorge and G. Binnig, Science 260, 1451 (1993).

${ }^{26}$ B. W. Hoogenboom, H. J. Hug, Y. Pellmont, S. Martin, P. L. T. M. Frederix,

D. Fotiadis, and A. Engel, Appl. Phys. Lett. 88, 193109 (2006).

${ }^{27}$ Q. Hong, M. F. Suárez, B. A. Coles, and R. G. Compton, J. Phys. Chem. B 101, 5557 (1997).

${ }^{28}$ G. T. Paloczi, B. L. Smith, P. K. Hansma, D. A. Walters, and M. A. Wendman, Appl. Phys. Lett. 73, 1658 (1998).

${ }^{29}$ H. H. Teng, P. M. Dove, and J. J. De Yoreo, Geochim. Cosmochim. Acta 63, 2507 (1999).

${ }^{30}$ R. Shiraki, P. A. Rock, and W. H. Casey, Aquat. Geochem. 6, 87 (2000).

${ }^{31}$ C. E. Jones, P. R. Unwin, and J. V. Macpherson, ChemPhysChem 4, 139 (2003).

${ }^{32}$ L. Wasylenki, P. Dove, D. Wilson, and J. De Yoreo, Geochim. Cosmochim. Acta 69, 3017 (2005).

${ }^{33}$ K. J. Davis, P. M. Dove, and J. J. De Yoreo, Science 290, 1134 (2000).

${ }^{34}$ J. J. De Yoreo and P. M. Dove, Science 306, 1301 (2004).

${ }^{35}$ G. Korosi and E. S. Kovats, J. Chem. Eng. Data 26, 323 (1981).

${ }^{36}$ V. Bouchiat and D. Esteve, Appl. Phys. Lett. 69, 3098 (1996).

${ }^{37}$ S. Hu, S. Altmeyer, A. Hamidi, B. Spangenberg, and H. Kurz, J. Vac. Sci. Technol. B 16, 1983 (1998). 